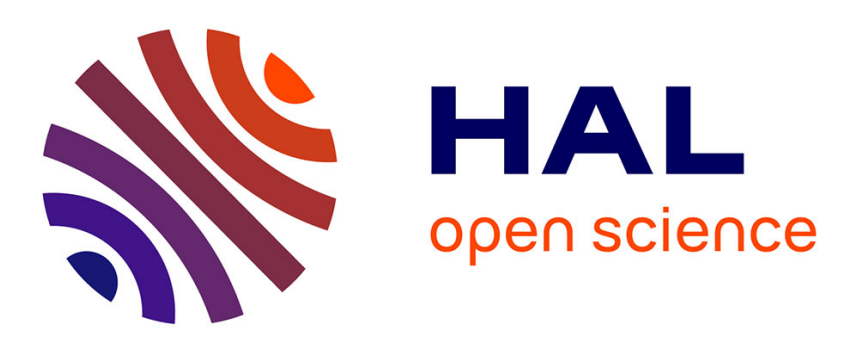

\title{
Stabilization of Collagen Fibrils by Gelatin Addition: A Study of Collagen/Gelatin Dense Phases
}

François Portier, Claire Teulon, Agnieszka Nowacka-Perrin, Flavien

Guenneau, Marie-Claire Schanne-Klein, Gervaise Mosser

\section{To cite this version:}

François Portier, Claire Teulon, Agnieszka Nowacka-Perrin, Flavien Guenneau, Marie-Claire SchanneKlein, et al.. Stabilization of Collagen Fibrils by Gelatin Addition: A Study of Collagen/Gelatin Dense Phases. Langmuir, 2017, 33 (45), pp.12916-12925. 10.1021/acs.langmuir.7b02142 . hal-01634652

\section{HAL Id: hal-01634652 \\ https://hal.sorbonne-universite.fr/hal-01634652}

Submitted on 14 Nov 2017

HAL is a multi-disciplinary open access archive for the deposit and dissemination of scientific research documents, whether they are published or not. The documents may come from teaching and research institutions in France or abroad, or from public or private research centers.
L'archive ouverte pluridisciplinaire HAL, est destinée au dépôt et à la diffusion de documents scientifiques de niveau recherche, publiés ou non, émanant des établissements d'enseignement et de recherche français ou étrangers, des laboratoires publics ou privés. 
Stabilization of collagen fibrils by gelatin addition: a study of collagen/gelatin dense phases

François Portier, ${ }^{a}$ Claire Teulon, ${ }^{b}$ Agnieszka Nowacka-Perrin, ${ }^{a}$ Flavien Guenneau, ${ }^{a}$ MarieClaire Schanne-Klein, ${ }^{b}$ and Gervaise Mosser ${ }^{*}, a$

a: Sorbonne Universités, UPMC Univ Paris 06, CNRS, Collège de France, LCMCP, F-75005 Paris France

b: LOB, Ecole polytechnique, CNRS, Inserm U1182, Université Paris-Saclay, F-91128 Palaiseau, France.

KEYWORDS: liquid crystal, self-assembly, collagen, gelatin, biomaterials

\begin{abstract}
.
Collagen and its denatured form, gelatin, are biopolymers of fundamental interest in numerous fields ranging from living tissues to biomaterials, food and cosmetic. This study aims at characterizing mixes of those biopolymers at high concentrations (up to $100 \mathrm{mg} \cdot \mathrm{mL}^{-1}$ ) at which collagen has mesogenic properties. We use a structural approach combining polarizationresolved multiphoton microscopy, polarized light microscopy, magnetic resonance imaging and transmission electron microscopy to analyze gelatin and collagen/gelatin dense phases in their
\end{abstract}


sol and gel states from macroscopic to microscopic scale. We first report the formation of a lyotropic crystal phase of gelatin A and show that gelatin must structure itself in particles to become mesogenic. We demonstrate that mixes of collagen and gelatin phase-segregate, preserving the setting of the pure collagen mesophase at gelatin ratio up to $20 \%$, and generating a biphasic fractal sample at all tested ratios. Moreover, differential scanning calorimetric analysis shows that each protein separates into two populations. Both populations of gelatins are stabilized by the presence of collagen, while only one population of collagen molecules is stabilized by the presence of gelatin, most probably those at the interface of the fibrillated microdomains and of the gelatin phase. While further studies are needed to fully understand the involved mechanism, these new data should have a direct impact on the bioengineering of those two biopolymers.

\section{INTRODUCTION.}

Collagens and gelatin are biopolymers widely encountered in various fields ranging from biomaterials, pharmaceuticals, food, photography etc. Those two proteins are intimately connected, the latter being the degradation product of the former.

In particular, Type I collagen, a fundamental structural brick of extracellular matrices of most of our tissues connective tissues (cornea, bone, tendon, skin, lung, blood vessel wall and cartilage $)^{1,2,3}$ presents various properties and structures ranging from the soft, flexible and transparent cornea, to the opaque, hard and mineralized bones. ${ }^{1,2,3}$ Due to its ubiquity, it is widely used in the field of biomaterials. ${ }^{4,5,6}$ Type I collagen is characterized by a peptide sequence of around one thousand amino acids, called $\alpha$ chain, built on a repetition of a triplet of 
amino acids "Glycine-XY". Type I collagen is rich in imino-acids (proline and hydroxyproline) and composed of about $15 \%$ ionizable amino acids. Three $\alpha$ chains fold into a right super triple helix ${ }^{1,2}$ by placing all glycine residues in the core of the helix and exposing other residues to the surface, thus promoting their interaction with the solvent or other molecules, including collagen. ${ }^{7}$ From a physico-chemical point of view, collagen is an amphoteric polyelectrolyte ${ }^{8,9}$ and its conformation relies on its net charge. The latter determines its further association into fibrils $7,10,11,12,13$ and, together with its concentration, its growth into more or less complex scaffolds. ${ }^{14,15,16,17}$

Gelatin is the product of irreversible thermal degradation of collagen in its $\alpha$ chains or in fragments. Nowadays, gelatin is omnipresent in our society with a production of around 350,000 tons per year (web site http: www.gelatine.org/fr/applications/gelatin.html). This protein is part of a large number of food products (sweets, pastries, dairy products, charcuterie...). It is used in pharmaceutical and medical fields (soft and hard capsules, tablets, haemostatic sponges, etc.). It is still used extensively in photography to produce ultra-sensitive films or glossy photo paper. ${ }^{18}$ It can also be combined with inorganic elements to give hybrid materials with specific properties. ${ }^{19,20}$ At low temperature, the local reformation of triple helix domains is responsible for the gelation of gelatin. ${ }^{21}$ This gelation depends on concentration, polydispersity of chains, temperature, cooling rate, etc. ${ }^{22,23,33}$

The high chemical resemblance of gelatin to collagen is at the root of its strong popularity in the field of biomaterials ${ }^{24,25,26}$ although it does not possess the hierarchical self-assembling properties of collagen ${ }^{7}$ and, to our knowledge, no liquid-crystal phases of gelatin solutions have been reported up to now. On the contrary, it has been shown that collagen solutions can selforganize at high protein concentration and form lyotropic mesophases, which structures are 
similar to those of in vivo tissues..$^{27,28,29,14,30,31,32,33}$ These collagen liquid phases are then stabilized by auto-association to obtain fibrillated biomaterials that are structured at different scales like connective tissues and exhibit the same high concentration of collagen (100 mg.mL ${ }^{-1}$ or even more). ${ }^{30,34,35}$ However, the large-scale use of collagen mesophases is hampered by the high price of collagen I and its low availability as a native protein. ${ }^{36}$

In this present study, we were interested to circumvent this drawback by checking the lyotropic properties of gelatin and evaluating the possibility to replace collagen by gelatin in the synthesis of biomaterials that exhibit high protein concentration and specific structures as in native connective tissues. To that end, we perform a multi-scale characterization of collagen/gelatin dense phases, in the liquid and solid states (ie. after fibrillogenesis) and demonstrate a new class of hierarchically structured materials, based on collagen/gelatin interactions set at interfaces of fibrillated micro domains.

\section{MATERIALS AND METHODS.}

Collagen Purification. Collagen was extracted from rat-tail tendons and purified as already described, using dissolution in acetic acid $(0.5 \mathrm{M})$, sodium chloride salt precipitations and centrifugations cycles. ${ }^{34}$ The final collagen solution was centrifuged for $3 \mathrm{~h}$ at $45,000 \mathrm{~g}$ and stored at $4^{\circ} \mathrm{C}$. The presence of protein contaminants was revealed by gel electrophoresis (bands at 46, 55 and $66 \mathrm{kD}$, data not shown). We did not check for non-protein contaminants. Acid composition of the collagen solution was modified by dialysis against the desired solvent. The collagen concentration was determined by assessing the amount of hydroxyproline. 


\section{Preparation of Gelatin Diluted Solutions and Fluorescein Isothiocyanate (FITC)-tagged}

Gelatin. Commercial gelatin A from porcine skin (Sigma-Aldrich, ref. G2625, 300 g bloom) was dispersed at $0.5 \%(\mathrm{w} / \mathrm{w})$ in acetic acid $(0.5 \mathrm{M})$ and warmed $15 \mathrm{~min}$ at $45^{\circ} \mathrm{C}$ to facilitate its solubilization. The solution was then filtered ( 0.2 microns), dialyzed intensively against the desired solvent and stored at $4^{\circ} \mathrm{C}$. Gelatin concentration in acidic solutions was determined by assessing the amount of hydroxyproline. The initial composition of each gelatin solution studied in glass-microchambers is reported in the supporting information (Table S1). (FITC)-tagged gelatin was prepared as reported in SI, following the protocol of Zou et al. ${ }^{37}$

Concentration of Collagen/Gelatin Solutions in Glass-Microchambers. Collagen/gelatin diluted solutions were obtained by mixing in the desired proportions diluted solutions of pure collagen (g0\%) and gelatin (g100\%) prepared separately as described above. The initial composition of each solution is reported in the supporting information (Table S2). Glassmicrochambers are two-sided open devices, which enable protein concentration through evaporation (Figure S2 of the supporting information), as reported previously. ${ }^{38}$

Preparation of Collagen/Gelatin Matrices. Collagen/gelatin diluted solutions prepared as

described above were concentrated up to $100 \mathrm{mg} \cdot \mathrm{mL}^{-1}$ by centrifugation-filtration using a commercial device (Vivaspin 30 KD) on a variable angle Beckmann centrifuge operating at 4000 g, $20^{\circ} \mathrm{C}$. The collagen/gelatin dense solutions were then cast in nuclear magnetic resonance (NMR) tubes (10 mm diameter) and fibrillated under ammonia vapor (overnight). Compositions of each collagen/gelatin matrix, and techniques used for their characterization, are reported in the 
supporting information (Table S3). Collagen/gelatin matrices were then washed and stored in deionized water at $4^{\circ} \mathrm{C}$. For each matrix, we determined proteins concentration by assessing their amount of hydroxyproline.

Sample preparation for microscopy. Collagen/gelatin matrices, prepared for Magnetic Resonance Imaging (MRI), were fixed in glutaraldehyde (2.5\%) and paraformaldehyde (8\%) before post fixation in osmium tetraoxide (2\%). The samples were then dehydrated through successive ethanol baths, included in araldite and sectioned into $1 \mu \mathrm{m}$ thin sections for optical microscopy (Polarized Second Harmonic Generation microscopy (P-SHG) and Polarized Light Microscopy (PLM)) and into $70 \mathrm{~nm}$ ultrathin sections for Transmission Electron Microscopy (TEM). The sections were stained with toluidine blue for PLM and with uranyl acetate for TEM. No staining was required for P-SHG.

Magnetic Resonance Imaging (MRI). MRI images were acquired on a $7 \mathrm{~T}$ Bruker Avance spectrometer, equipped with $2 \mathrm{~T} \mathrm{~m}^{-1} \mathrm{X}, \mathrm{Y}$ and $\mathrm{Z}$ gradients and a $10 \mathrm{~mm}$ micro imaging probe, using Rapid Acquisition with Relaxation Enhancement (RARE) pulse sequence. Echo time was set to $60 \mathrm{~ms}$ and the RARE factor to 8. In 2D experiments, the slice thickness was set to $200 \mu \mathrm{m}$ and 128 repetitions were accumulated, with the repetition delay of $3 \mathrm{~s}$. Image resolution of $49 \mathrm{x}$ $49 \mu \mathrm{m}$ was obtained with $256 \times 512$ points and FOV $12.5 \times 25 \mathrm{~mm}$. The 3D dataset was acquired with 16 repetitions and an 80.9 s repetition delay. 256 x 512 × 256 points were acquired in the FOV of 12.5 x $25 \times 12.5 \mathrm{~mm}$, resulting in the resolution of $49 \times 49 \times 49 \mu \mathrm{m}$.

Light Microscopies. Samples were probed by multiphoton microscopy using a custom-built 
setup with 60x, 1.2 NA objective as previously described. ${ }^{39}$ We recorded two modes of contrast: (i) Second Harmonic Generation (SHG), which is specific to aligned and polarized collagen triple helices (or collagen fibrils) and shows no signal from centrosymmetric molecular alignments, and (ii) two-Photon Excited Fluorescence (2PEF), which probes intrinsic fluorescence signals, or FITC-labeled gelatin. ${ }^{39,40,41}$ Circular polarization was used to mitigate orientation effects in the image plane and linear polarizations with different orientations (P-SHG) were used to reveal the main orientation of collagen structures. ${ }^{34}$ Imaging of microchambers was performed within 2 hours after stopping the injection to prevent sample dehydration. ${ }^{39}$ Samples were also analyzed by PLM as, unlike SHG, PLM is equally sensitive to centro or non-centro symmetric molecular organizations. Between crossed polarizers, dilute isotropic collagen solutions appeared black, while colored light appeared for concentrated solutions due to the birefringence of their liquid crystal organization. We used a Nikon Eclipse E600Pol microscope equipped with a 40x/0.65 NA objective and a DXM 1200 CCD camera. Both for SHG and PLM imaging, microchambers were set horizontally for observations of the fluid state.

Transmission Electron Microscopy (TEM). Ultrathin sections of glutaraldehyde-fixed and araldite-embedded samples were observed on a FEI Tecnai spirit G2 operating at $120 \mathrm{kV}$ and images were recorded on a CCD Camera (Orius Gatan 832 digital).

Differential Scanning Calorimetry (DSC). DSC thermograms of dense collagen/gelatin matrices were recorded in a DSC Q20 (ThermoAnalytic) at $5^{\circ} \mathrm{C} / \mathrm{min}$ heating rates. Each sample was freshly cut, weighted and encapsulated in a hermetic aluminum pan prior to a stabilization step at $5^{\circ} \mathrm{C}$. For each condition, 4 samples were analyzed. Each thermogram obtained by DSC 
was deconvoluted: a least squares method was used to fit the thermograms by the sum of four Gaussian functions (corresponding to the proteins denaturation peaks), and a sigmoid function (reflecting a change in the specific heat of the matrix during the denaturation process). The denaturation temperatures are defined as the position of the peak obtained for the Gaussian functions. The specific enthalpies are defined as the area of the Gaussians functions divided by the protein (collagen or gelatin) dry weight and the heating rate $\left(5^{\circ} \mathrm{C} / \mathrm{min}\right)$.

\section{RESULTS AND DISCUSSION.}

\section{Liquid crystal organization in concentrated solutions.}

\section{Pure Gelatin.}

After a few days of continuous injection into a glass-microchamber, gelatin forms a dense phase at the air/water interface that exhibits a strong and homogeneous birefringence when observed with Polarized Light Microscopy (PLM). Birefringence signature was observed for each condition of $\mathrm{pH}$ and acid type (Table S1 of the supporting information). In all conditions, near the interface or opposite to the injection site that corresponds to high gelatin concentrations, we observe gelling of the gelatin mesophase (Figure 1). Increasing the gelatin initial concentration from 0.2 to $2 \mathrm{mg} \mathrm{mL}^{-1}$ increases the volume of this gel phase. When observed with Second Harmonic Generation microscopy (SHG), gelatin mesophase does not exhibit any SHG signal. 


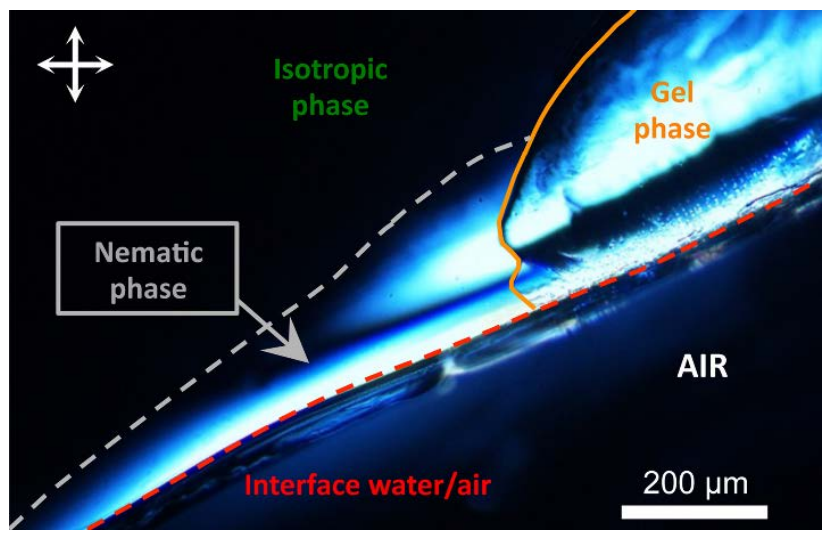

Figure 1. Gelatin A dense phase (condition 6, see SI) observed by PLM (cross-polarizer represented by the white arrows) after 2 weeks of continuous injection in the microchamber. At the water/air interface (red dotted line), we could notice the presence of a liquid and homogeneously birefringent phase (delineated by the grey dotted line), a characteristic pattern of the nematic mesophase. We also observed the gelation of the mesophase at higher concentration (dry phase delineated by orange line).

Observation of gelatin dense phase by PLM microscopy demonstrates that gelatin can form lyotropic mesophases in acidic solution (Figure 1 and Figure S1). This is the first report of a gelatin A liquid crystal phase. To obtain information on the mesogen nature, gelatin mesophases were thermally denatured using a mild thermal treatment with a very limited probability of chain hydrolysis. We observe an initial loss of birefringence, which demonstrates the crucial role of triple helices segments in the formation of the liquid crystal phases. This is confirmed by the observation that the liquid crystal phase forms again after few hours, due to the reversibility of the denaturation process of triple helical segments in gelatin. Three hypotheses can be made 
about the gelatin mesogen: it may be the unfolded gelatin $\alpha$ chain (1), triple helical segments (2) or a gelatin "particle" (3). The loss of birefringence above the triple helix denaturation temperature implies that this mesophase depends on triple helix formation, which rules out the first hypothesis. Moreover, Guo et al. demonstrated that triple helix nucleation is directly linked to the $\alpha$ chain concentration so that, in dense gelatin solutions, proportion of unfolded gelatin chain is expected to be very low. ${ }^{42}$ Helices are chiral objects generating chiral nematic phase, ${ }^{43}$ while we never observe such phases with gelatin, meaning that triple helices cannot constitute the mesogen by themselves, ruling out the second hypothesis.

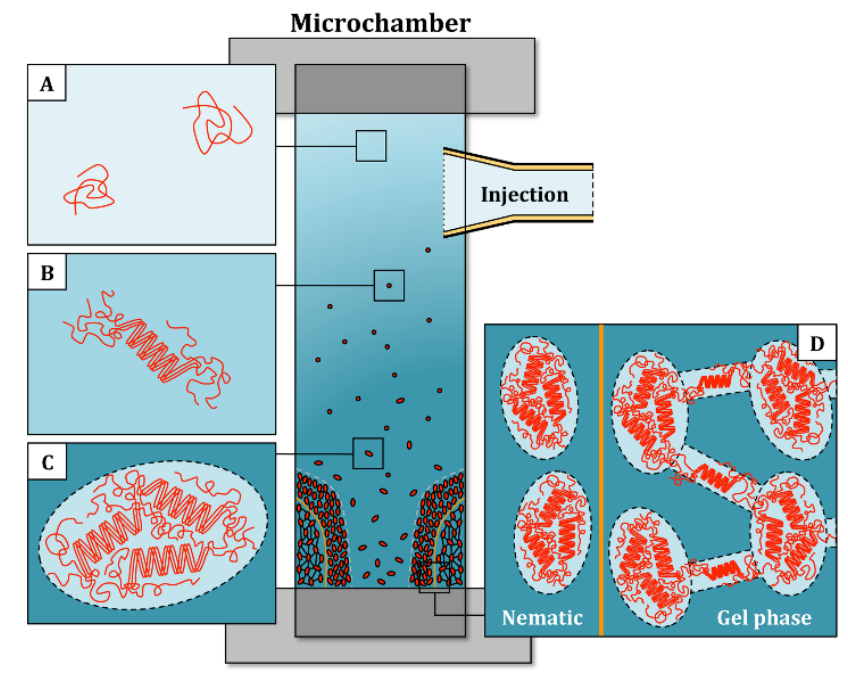

Figure 2. Formation of the gelatin mesophase in a microchamber (top view). An acidic and diluted solution of gelatin A $\left(0.2 \mathrm{mg} \cdot \mathrm{mL}^{-1}\right)$ is injected in the microchamber. Close to the injection site, we essentially find single gelatin chains with few triple helix segments (A). Away from this site, gelatin concentration increases gradually, promoting the formation of triple helices (B) and then anisotropic particles (C), with no preferred polarity. At higher concentration, those particles could assemble in nematic mesophases (D). Finally, in the more concentrated areas, triples helices bridge the particles and induce a gelation. 
Therefore, the next hypothesis is that the mesogen would be a particle resulting from local aggregation of gelatin triple helices. The birefringence of gelatin dense phases implies that gelatin particles are anisotropic and constituted of aligned triple helix segments. The absence of SHG signal from the gelatin mesophases further indicates that the mesogen is centrosymmetric. Consequently, the relative arrangement of gelatin helices, within the particle, must be centrosymmetric and anisotropic, i.e. aligned along a main direction, without a preferred polarity (anti-parallel averaged arrangement). We believe that, within a particle, triple-helix segments are separated by unfolded ones, which restrain the movement of the formers and hampers their polarized association. Figure 2 schematizes the mesophase formation integrating the generation of gelatin particles. To support this hypothesis, it should be noted that the formation of gelatin particles (also named aggregates) have already been reported in a different context and at low concentration in gelatin. ${ }^{44}$

Finally it should be noted that similarly to collagen ${ }^{45,38}$ gelatin mesophases appear to be affected by $\mathrm{pH}$ or acid composition, at least at the scale of our observations (Figure S3). This indicates that the gelatin mesogen is sensitive to $\mathrm{pH}$ and acid type besides being sensitive to temperature. Consequently, highly controlled experimental conditions would be required for the observation of these fragile phases in a reproducible manner and to study them more thoroughly.

\section{Pure Collagen and mixes of collagen and gelatin.}

The collagen dense phase prepared in $10 \mathrm{~mm}$ acetic acid and $0.3 \mathrm{~mm}$ hydrochloric acid exhibits an intense birefringence with a dark and bright alternating pattern that extends up to several hundred of micrometers in the glass-microchamber (Figure 3). The transitions between dark and 
bright lamellas are abrupt as those of a plywood phase. This structure has been validated by PSHG and numerical calculations. ${ }^{39}$

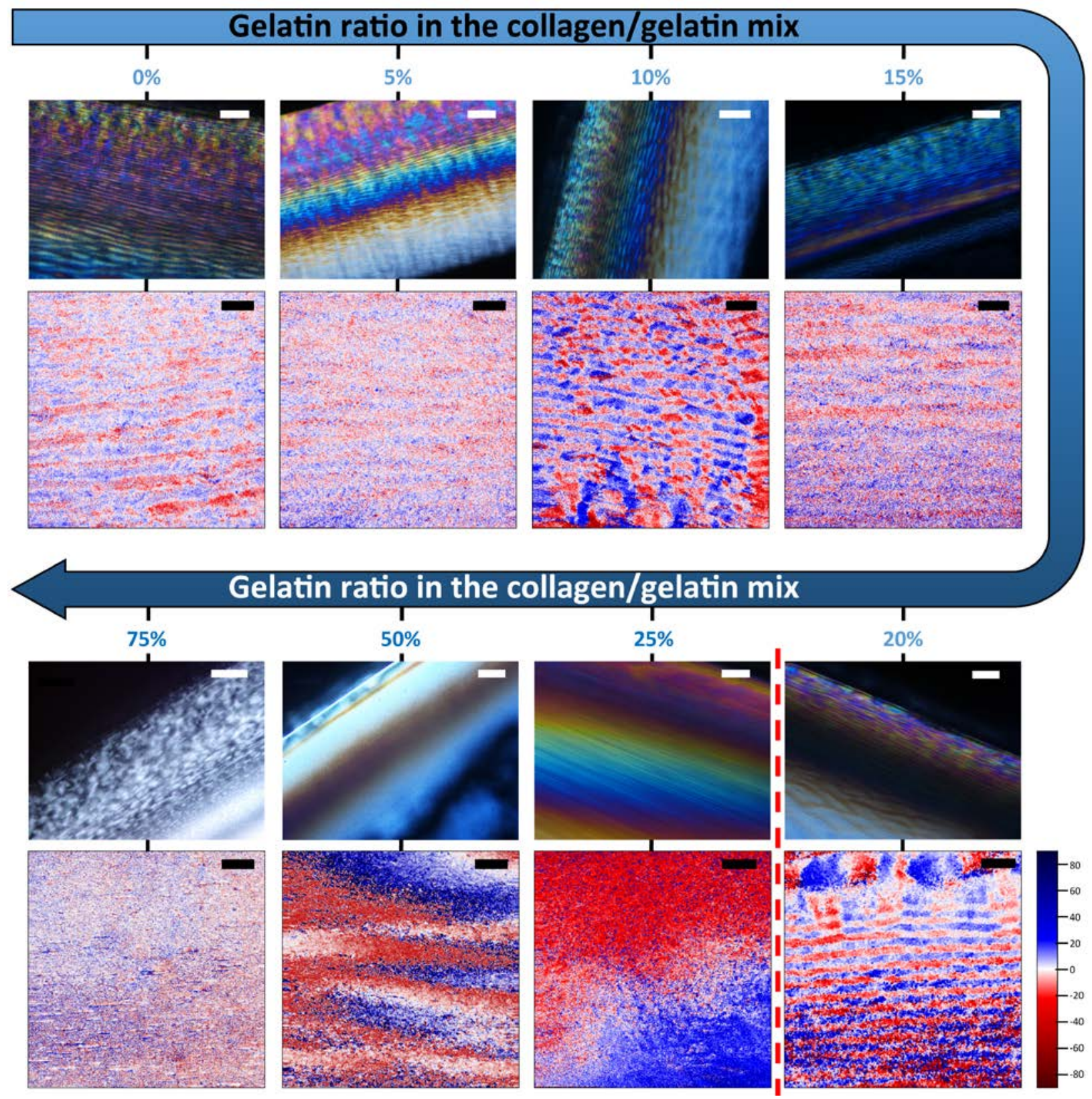

Figure 3. Effect of gelatin addition on the formation of collagen mesophase. After 4 days of continuous injection, collagen/gelatin dense phases were observed by PLM (top lines) and PSHG (bottom lines, main orientation of collagen triple helices obtained from P-SHG images). The characteristic pattern of a plywood mesophase is present up to a gelatin ratio of $20 \%$. From 
$25 \%$ to $50 \%$ no clear textures are seen. From $75 \%$ upward, a birefringent liquid phase is observed with only a local and limited alignment of the collagen molecules. The scale bars are respectively 50 and $5.5 \mu \mathrm{m}$ for PLM and P-SHG orientation pictures.

PLM and P-SHG observations show that the mesophase organization observed with pure collagen is preserved up to a gelatin ratio of $20 \%$ (Figure 3). At 25\% and above the pattern is lost. For samples with $\mathrm{rgelatin}_{\mathrm{n}}>75 \%$, we observe dispersed droplets emitting strong SHG signals in a "dark", continuous phase (Figure 4A). PLM observations show that all samples exhibit a strong birefringence (Figure S4). (FITC)-tagged gelatin A is used to investigate the composition of the two phases (Figure 4B and 4C). It shows that areas of low fluorescence intensity, which are devoid of gelatin, are co-localized with SHG signal corresponding to collagen.

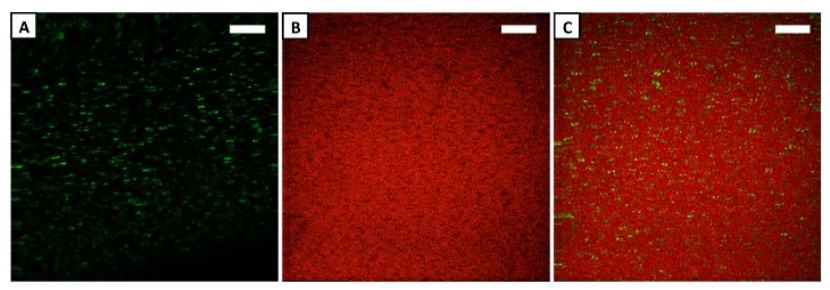

Figure 4. Collagen/Gelatin phase separation. At high gelatin ratio $\left(\mathrm{r}_{\text {gelatin }}=85 \%\right)$ and high protein concentration, droplets emitting an intense SHG signal are dispersed in a "dark" continuous phase (4A, observed by SHG). Collagen/(FITC)-tagged gelatin mix (15\%-85\%) continuously injected in a microchamber for 4 days show that areas of low fluorescence intensity (4B, observed by 2PEF) are colocalized with strong SHG signal (4C, 2PEF: red, SHG: green). Scale bars: $10 \mu \mathrm{m}$.

The samples at high gelatin ratio ( $\mathrm{r}_{\text {gelatin }}>75 \%$ ) thus present a phase segregation characterized by dispersed droplets of collagen with strong SHG signal within an otherwise "dark" continuous 
phase of gelatin. We hypothesize that this collagen/gelatin phase separation also occurs in all other collagen/gelatin mixes, since this is observed in matrices (see below) while it is known that collagen fibrillogenesis does not alter the initial liquid organization. ${ }^{14}$ This phase separation can explain the sudden loss of the collagen plywood structure observed for $\mathrm{r}_{\text {gelatin }}>20 \%$. At low gelatin ratio ( $\mathrm{r}_{\text {gelatin }}<20 \%$ ), the gelatin is dispersed in the continuous phase of collagen and may slightly perturb the formation of the plywood structure. At those ratios, we suppose that the gelatin is essentially present in liquid-crystal defects. Above a gelatin ratio of $20 \%$, the gelatin phase becomes continuous and prevents formation of collagen plywood structure while collagen locally assembles in nematic polarized domains. Since the surrounding gelatin phase shows birefringence like pure gelatin, we suggest that, here also, gelatin forms a nematic mesophase.

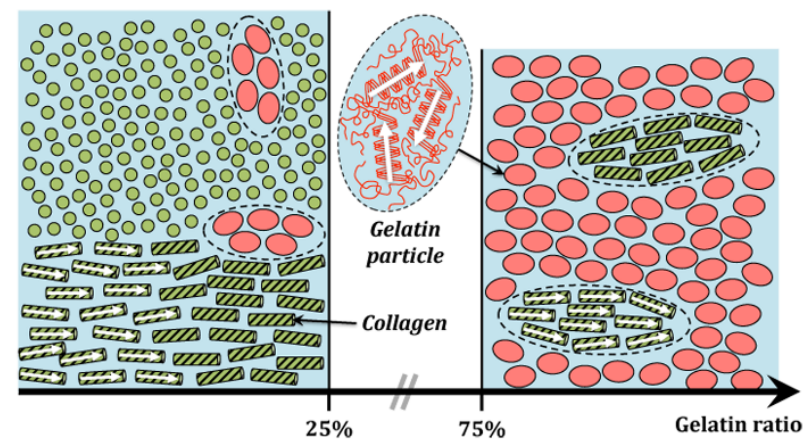

Figure 5. Phase diagram of the Collagen/Gelatin dense liquid phases. White arrows indicate the polarity of the triple helices ( $\mathrm{N}$ to $\mathrm{C}$ direction).

Figure 5 displays the structure of collagen/gelatin mixes per gelatin ratio inferred from our results. Several hypotheses may explain the collagen/gelatin phase separation: (1) Phase separation has been already described for thermotropic mesogen mixes that form mesophases of different symmetries. ${ }^{43}$ However, this symmetrical cause cannot be retained here because both collagen and gelatin forms (chiral) nematic phases. (2) From a thermodynamic point of view, the 
model generally used to interpret the miscibility of biopolymers is the Flory-Huggins lattice model. ${ }^{46}$ This approach has been used to model the behavior of polymer mixtures and ternary, biopolymer/solvent systems. The model is essentially cast in terms of the relative molar volumes of the two biopolymers, here collagen and gelatin, and a set of so-called $\chi$ parameters that are related to the interaction of the biopolymers with the solvent $(\chi$ collagen-water and $\chi$ gelatin-water $)$ and

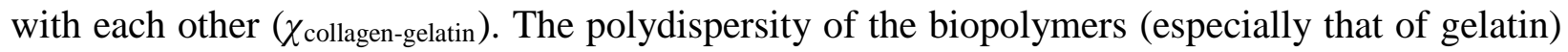
can impact the interactions between the two polymers. ${ }^{47}$ Any perturbation of any of these parameters may modify the mixture thermodynamics and induce a phase separation. In our system, at $20^{\circ} \mathrm{C}$ and high protein concentration, gelatin is mainly composed of triple helix segments, which are similar to collagen molecules from chemical and structural points of views. This means that the progressive formation of triple helices of gelatin during the concentration process should favor the miscibility of the two polymers. However, these ordering and autoassociation of gelatin also results in entities of increased molecular weight and polydispersity; these effects are probably the main driving forces behind the phase separation. (3) We report here that gelatin forms lyotropic mesophases and we suppose that the mesogens are gelatin particles stabilized by triple helices segments. A collagen/gelatin mix, at high concentration, shows consequently polydisperse gelatin particles of high molecular weight surrounded by semiflexible rods of collagen. ${ }^{48}$ According to the "depletion model", collagen molecules at the vicinity of gelatin particles encounters a decrease of their configurational entropy, which results in a repulsive interaction. ${ }^{49}$ This kind of interaction is essentially of entropic nature so that it can be observed even with proteins that are chemically and structurally similar. In addition, this depletion model has already successfully explained the phase separation between gelatin and dextran. ${ }^{50}$ In this work, Edelman et al. conclude that gelatin association in clusters (here 
particles) enhances the phase separation due to depletion, which fits well with the observation in our systems.

\section{Fibrillogenesis and Matrices.}

After fibrillogenesis, matrices are characterized at different scales: microscopic by Transmission Electron Microscopy (TEM), mesoscopic by SHG and PLM and macroscopic by Magnetic Resonance imaging (MRI) (Figure 6). The relation between collagen and gelatin into hybrid matrices is analyzed by Differential Scanning Calorimetry (DSC) (Figures 7 and 8).

Pure Collagen and Gelatin Matrices (m0\%, $m 100 \%$ ).

Pure collagen matrices (m0\%), at microscopic scale, are formed of a dense network of locally organized fibrils (Figure 6A). At mesoscopic scale, samples are composed of birefringent areas, with an organization similar to a banded structure, surrounded by a non-birefringent matrix (Figure 6B). Those mesoscopic heterogeneities are also observed by SHG, where both low and high SHG intensity areas are found. At macroscopic scale, pure collagen matrices exhibit also a heterogeneous structure when imaged with MRI (Figure 6C). In contrast, pure gelatin matrices (m100\%) appear homogeneous with no structuration at any scale (Figure 6M-O). 


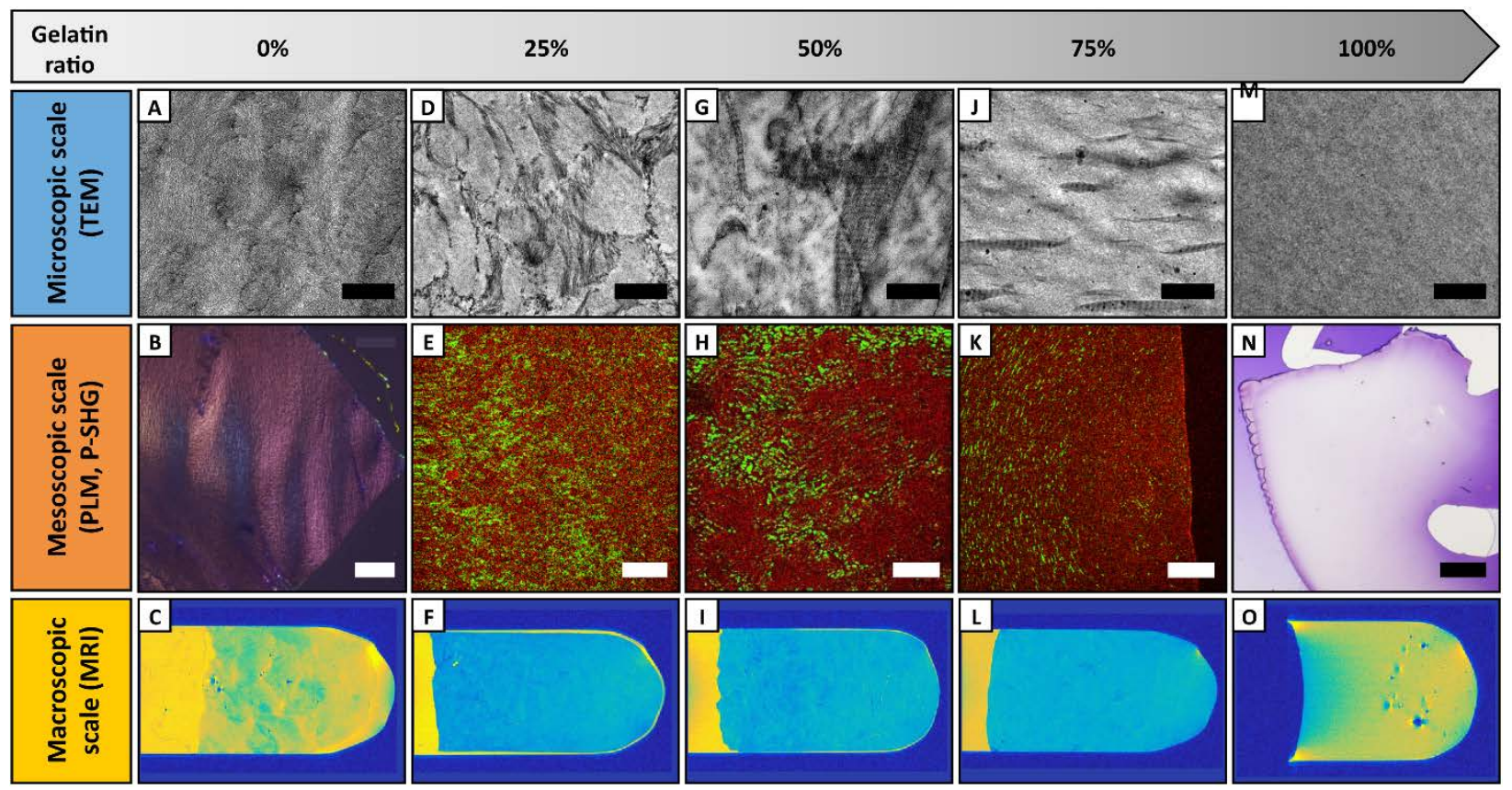

Figure 6. Collagen/Gelatin dense matrices. Collagen/Gelatin matrices were observed from

microscopic to macroscopic scales using complementary techniques: TEM (A, D, G, J M), PSHG (E, H, K), PLM (B, N) and MRI (C, F, I, L, O). On P-SHG pictures, SHG is displayed in green and 2-PEF in red. A detailed description of those pictures is found in the text. TEM and SHG scale bars: $1 \mu \mathrm{m}$ and $75 \mu \mathrm{m}$ resp. Extern diameter of the MRI tube: $10 \mathrm{~mm}$.

Hybrid Matrices (m25\%, m50\% and $m 75 \%$ ).

At microscopic scale, micrometric domains of aligned fibrils, exhibiting the characteristic 67 nm D-period of native fibrillated collagen, are observed in hybrid matrices (Figure 6D, 6G, 6J). In $\mathrm{m} 25 \%$ and $\mathrm{m} 50 \%$, the domains are interconnected to each other (Figure 6D, 6G), while in m75\% domains are isolated in an amorphous matrix (Figure 6J). At mesoscopic scale, aligned and polarized collagenous domains are identified by their ability to emit strong SHG signal (in green on the Figure 6E, 6H, 6K). Those domains are heterogeneously distributed in the samples. 
We observe two kinds of areas: areas with low and areas with high density of collagen. MRI reveals heterogeneous signal distribution within the matrices (Figure 6F, 6I, 6L).

TEM, P-SHG and MRI thus reveal that fibrillogenesis of concentrated collagen/gelatin mixes results in heterogeneous materials with a multi-scale structure. In most collagen/gelatin matrices, collagen triple helices are associated in thin fibrils. Those are locally aligned and form microdomains, which exhibit the characteristic collagen $67 \mathrm{~nm}$ D-period (TEM). Those microdomains (SHG) are heterogeneously dispersed in a gelatin matrix (no SHG) and we can distinguish, at macroscopic scale, areas with high and low content of organized domains (MRI). Thus, there is an overall hierarchical organization of collagen/gelatin resembling a fractal. This phase separation that takes place in the liquid state and is further stabilized by the $\mathrm{pH}$ increase, is very complex. It would be interesting to look at the evolution of this phase separation over time. However, due to the high viscosity achieved at those concentrations or to the gelling of those polymers $^{51,52}$ this was not considered in the course of this work.

Heterophase systems generated by gelatin in presence of other polymers such as polyethyleneglycols or polysaccharide have already been reported. ${ }^{53,46,54}$ In those systems, phase segregation was stopped by the gelatin gelling, stabilizing by this way the initial water-in-water emulsion. ${ }^{47,55}$ We think that such mechanism could also take place in the case of collagen/gelatin mixes at high concentration. Indeed, even if the $\mathrm{pH}$ of our solution is quite far from the $\mathrm{pI}$ of gelatin A, we observed nevertheless gelatin gelling in microchambers at highest concentrations near the air/water interface (Figure 1). Consequently, even at low pH, the progressive gelling of the gelatin, associated with the high viscosity of the solution, might prevent the complete demixing of collagen and gelatin phases. 
Similarly to our collagen/gelatin mixes, Norton et $a .^{56}$ also demonstrated that water-in-water emulsion morphology was directly related to the polymer ratio: from a simple mix of gelatin and maltodextrin in water, one obtain a direct emulsion at high gelatin ratios, an inverse emulsion at low gelatin ratios or a bicontinuous system for an equivalent quantity of both polymers.

The singularity of our system relies on the use of the same protein, in its native and denatured form, in order to generate a water-in-water emulsion and then a bigel (or co-gel). In addition, it is also the first example of bigel constituted by stabilized mesophases. The use of such organized domains improve the hierarchical structure of our materials and, so, their ability to mimic the invivo tissues.

\section{Collagen and Gelatin Interaction in Hybrid Matrices.}

The denaturation processes of fibrillated collagen and gelatin are studied by DSC at all collagen/gelatin ratios (Figure 7 \& Figure S5). Thermograms of the pure proteins present a single peak relative to the triple helix denaturation (Figure 7A). We observe that denaturation of the fibrillated collagen occurs at higher temperature than for gelatin as already reported. ${ }^{45}$ Thermograms of hybrid collagen/gelatin matrices (m5\% to m95\%) present two endothermic peaks, one at "low" temperature $\left(35^{\circ} \mathrm{C}\right.$, gelatin) and one at "high" temperature $\left(50^{\circ} \mathrm{C}\right.$, fibrillated collagen). Moreover, for the matrices with rich gelatin content (m75\% - m85\%), two peaks are clearly distinguished relative to the collagen denaturation (inset of Figure 7A): a peak aligned on the denaturation peak of the pure collagen matrix (around $49^{\circ} \mathrm{C}$ ) and a new peak, at higher temperature (around $53^{\circ} \mathrm{C}$ ). For each gelatin percentage, the data were deconvoluted as the sum of four Gaussian functions. Figure S5 shows the thermogram of a m85\% matrix together with the 
numerical fit and the four Gaussian functions. Those were used to determine the average temperatures and enthalpies of denaturation of the four entities (Figure 5B -E).

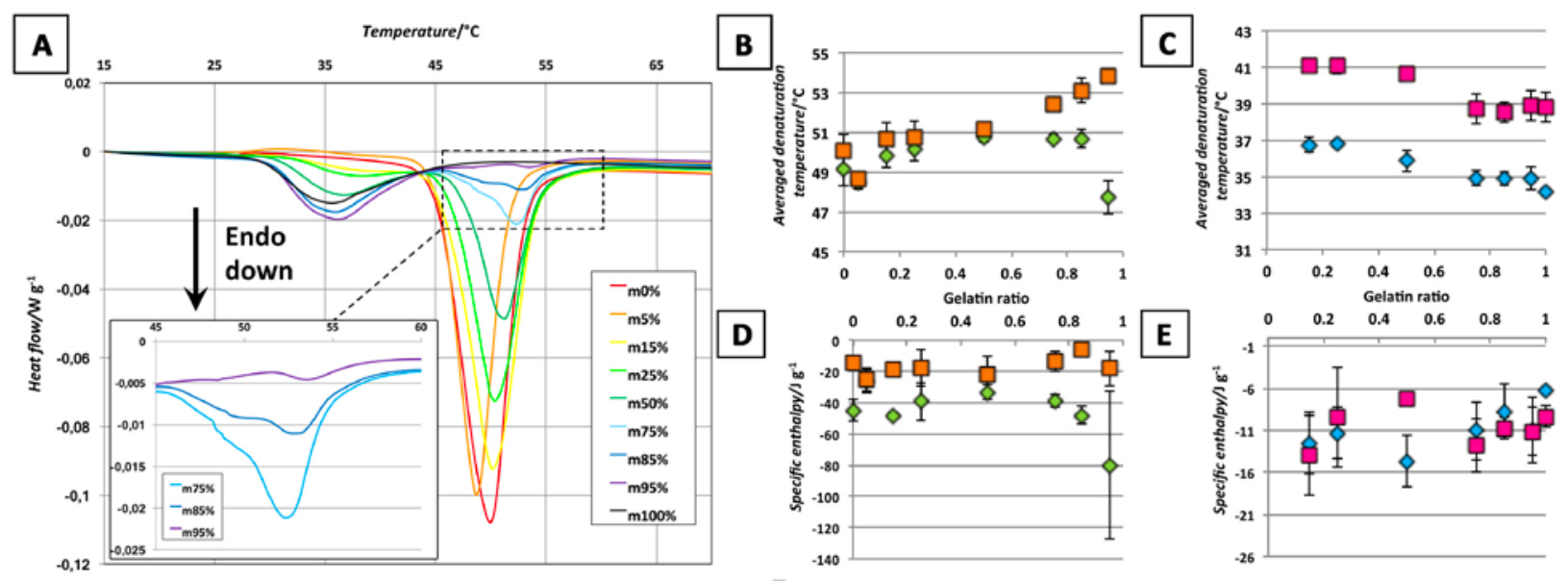

Figure 7. Analysis of the denaturation of the collagen/gelatin matrices by DSC. A) Thermograms of the Collagen/Gelatin dense matrices between 5 and $70^{\circ} \mathrm{C}$ at a rate of $5^{\circ} \mathrm{C} / \mathrm{mn}$. During the temperature increase we successively observed the denaturation of the gelatin triple helices (broad peak centered on $35^{\circ} \mathrm{C}$ ) and then of the fibrillated collagen ("sharp” peak centered on $50^{\circ} \mathrm{C}$ ). The matrices with a high gelatin ratio (inset) showed two peaks relative to the collagen denaturation (see also Figure S5). For one of the peak, the average collagen denaturation temperature increased with the gelatin ratio while it remained constant for the second one. B), D) and C), E) average temperatures and specific enthalpies of denaturation of fibrillated collagen and gelatin as a function of the gelatin ratio (measured from the deconvoluted peaks, see Figure S5 for details). Each value is an average on 4 samples. Error bar: Standard deviation. 
The average denaturation temperature of one of the fibrillated collagen deconvoluted peak increases with increasing gelatin ratio. (Figure 7B, orange marks). A maximum increase of $6^{\circ} \mathrm{C}$ is observed for $\mathrm{m} 95 \%$. The values of the second peak remain relatively constant (Figure 7B, green marks). Concerning the denaturation of gelatin we also observe two peaks indicative of two gelatin populations. However, in this case, the average denaturation of both populations is shifted when the concentration of collagen is increased (at low gelatin ratios). We observe a shift of $2.5^{\circ} \mathrm{C}$ for the denaturation temperature of gelatin for m25\% (Figure 7C, pink and blue marks). Collagen specific enthalpies of denaturation are quite stable for both collagen populations (Figure 7D, green and orange marks). The high error of the value found for $\mathrm{m} 95 \%$ makes this value unreliable. Regarding gelatin, the errors are quite large for both populations. However, we can tentatively consider that the gelatin specific enthalpies remain constant (Figure 7E pink and blue marks). For m5\%, specific enthalpies are too low to be precisely measured.

The two peaks found on these DSC thermograms of collagen/gelatin matrices reveals the presence of two populations of fibrillated collagen and of gelatin. Moreover, the different behavior of the two populations of fibrillated collagens when the temperature increases indicates that one population is sensitive to the presence of gelatin and the other not. The increase of the average denaturation temperature of the first population of collagen when the gelatin ratio increases further indicates that the presence of gelatin stabilizes this fibrillated collagen population. The non-effect of gelatin on the average denaturation temperature of the second population of fibrillated collagen suggests that this second population is not in contact with gelatin. Based on those observations, we can comfortably hypothesize that: (1) the first population corresponds to collagen molecules on the periphery of either isolated collagen fibrils 
or of fibrillated collagen domains, (2) the second population very likely corresponds to collagen molecules either in the core of isolated fibrils or in the core of the fibrillated domains. We observe that the collagen specific enthalpy is constant when the gelatin ratio increases whereas denaturation temperature progressively increases. This means that the specific denaturation enthalpy of fibrillated collagen is not modified by its interaction with gelatin, so that this stabilizing interaction is probably of entropic nature. This is further sustained by the observation that the collagen enriched domains are getting smaller and smaller with increasing gelatin ratio (Figure 6H-K) resulting in an equal and homogenous distribution of the two entities throughout the sample.

Concerning the gelatin, our data indicate that there are two populations and that both of them seems equally affected by the presence of collagen. At this stage, we can make two hypothesis: (1) the first population is composed of triple helices of gelatin within the gelatin particles and the second population are triple helices of gelatin in interaction with collagen whether at the periphery of particles or isolated, (2) the first population are isolated gelatin triple helices and the second population are triple helices from gelatin particles. The fact that their average temperature of denaturation equally decreases with increasing gelatin ratio (i.e. increases when increasing the proportion of collagen) suggests that the interactions of both entities in regards to collagen are the same. This last observation is more in favor of the second hypothesis and further indicates that the nature of the interactions between the gelatin triple helices and the fibrillated collagen is rather the same, whether gelatin triple helices form a particle or not. We can hypothesize that the interactions taking place here rely on the water networks, which play an important role in stabilizing the two proteins, from their helical structures to their intermolecular organization. ${ }^{57}$ 
Yamashita et al. have reported a similar increase of the gelatin denaturation temperature consecutive to the addition of low molecular weight polyethyleneglycols. ${ }^{53}$ However, as in our case, the molecular origin of this increase has not been determined. Interestingly, the quantity of polyethyleneglycols in their solution was not high enough to induce a phase segregation that moreover stabilizes both proteins. This suggests that it may be possible to achieve, with an appropriate composition ( $\mathrm{pH}$, protein concentration, gelatin ratio, type of acid, ionic force...) a homogeneous collagen/gelatin mix that would exhibit a mutual stabilization. Such mixtures will be valuable to further analyze the nature of the stabilizing collagen/gelatin interactions.

\section{SUMMARY AND CONCLUSIONS.}

Our structural study of gelatin, collagen and hybrid dense phases in sol and gel states provides new insight into gelatin properties and collagen/gelatin interactions: 1) We have reported the first lyotropic liquid crystal phase formed from gelatin A and shown that it can be affected by temperature, $\mathrm{pH}$ or acid composition. We have demonstrated that this gelatin mesophase relies on the formation of triple helix segments that are presumably organized into centrosymmetric anisotropic particles. 2) We have demonstrated that collagen/gelatin phase separation occurs at high protein concentration. At ratio up to $20 \%$ in gelatin, the collagen mesophase is not affected by the presence of gelatin. We observe biphasic fractal-like samples first enriched in collagen and then enriched in gelatin. At high gelatin ratio, this phase separation is at the origin of collagen nematic "droplets" or tactoids dispersed in the gelatin mesophase. 3) Multiscale characterization of resulting hybrid matrices shows that they are heterogeneous materials with complex and hierarchical structure of collagen. 4) Finally, differential scanning calorimetric 
analysis showed that both protein types could be separated into two classes: Collagen - (a) A first class of collagen molecules directly in contact with and sensitive to gelatin triple helix, most likely corresponding to collagen at the periphery of isolated fibrils of collagen fibrillar domains. This class is stabilized by interactions with gelatin (b) a second class of collagen in the core of the fibrils or in the core of collagen fibrillar domains that is not affected by the presence of gelatin. Gelatin - (a) A first class of gelatin triple helices, which constitute gelatin particles, (b) A second class of gelatin triple helices that are not involved in particles. Both classes of gelatin show identical interactions with collagen fibrils and are stabilized when interacting with fibrillar collagen. The interaction between collagen and gelatin are of entropic nature that likely involves the water network structuring the proteins.

Our results open up new routes for applications in living tissues, biomaterials, food and cosmetic as well as for the development of organic/inorganic materials. In the field of biomaterials, this new class of dense and fractal-like collagenous matrices could serve as a support for the synthesis of artificial organs presenting this type of organization as lung or spleen. Indeed, gelatin could either act as a porogen, while being removed from the hybrid phase, or represent a weaker path easing cells invasion. In a similar way, new drug delivery systems could be developed starting from these matrices. Since collagen and gelatin interact differently with inorganic elements, such biphasic-fractal like materials may also be a source of inspiration in the field of organic/inorganic materials..$^{19,58,59,60}$ Given that gelatin is a cheap product available in large quantities, all these new materials should find large-scale applications in the near future.

\section{ASSOCIATED CONTENT}


Supporting Information. Contents: composition of protein solutions and matrices, protocol for FITC-tagged gelatin synthesis, scheme of a microchamber and study of gelatin dense phase organization above gelatin denaturation temperature. The following files are available free of charge.

\section{AUTHOR INFORMATION}

\section{Corresponding Author}

*E-mail: gervaise.mosser@upmc.fr

\section{Author Contributions}

The manuscript was written through contributions of all authors. All authors have given approval to the final version of the manuscript. F.P., C.T., A.N.-P. and G.M. performed the experiments. C.T. and M.-C.S.-K. performed the numerical and analytical calculations for P-SHG. A.N.-P. and F.G. analyzed the MRI data. F.P. analyzed the DSC experiments. F.P., C.T., A.N.-P., F.G., M.-C.S.-K. and G.M. discussed the data in terms of collagen and gelatin organization in sol state and material structure in gel state. F.P., G.M. and M.-C.S.-K. wrote the manuscript. G.M. conceived the project.

\section{ACKNOWLEDGMENT}

The authors thank Marie-Christine Heng for her technical support during her internship, Bernard Haye (LCMCP) for the preparation of the TEM samples, Francisco Fernandes, François Ribot and Aurélien Tidu (LCMCP) for their fruitful discussions and the reviewers for their stimulating questions. This work was partly supported by the Fondation pour la Recherche Médicale (Grant No: DCM201221225759) and by the Agence Nationale de la Recherche (ANR10-INBS-04 and ANR-11-EQPX-0029). F.P. was supported by a grant of the Ecole doctorale 
397 (Physique et Chimie des Matériaux) and the UPMC. C.T. was supported by a grant "Contrat

Doctoral Spécifique Normalien” from MESR.

\section{REFERENCES}

(1) Kadler, K. E.; Baldock, C.; Bella, J.; Boot-Handford, R. P. Collagens at a Glance. J. Cell Sci. 2007, 120 (12), 1955-1958.

(2) Collagen: Structure and Mechanics; Fratzl, P., Ed.; Springer: New York, NY, 2008.

(3) Prockop, D., J. What Holds Us Together? Why Do Some of Us Fail Apart? What Can We Do About It? Matrix Biol. 1998, 16, 519-528.

(4) Parenteau-Bareil, R.; Gauvin, R.; Berthod, F. Collagen-Based Biomaterials for Tissue Engineering Applications. Materials 2010, 3 (3), 1863-1887.

(5) Sionkowska, A.; Skrzyński, S.; Śmiechowski, K.; Kołodziejczak, A. The Review of Versatile Application of Collagen: Versatile Application of Collagen. Polym. Adv. Technol. 2017, 28 (1), 4-9.

(6) Miranda-Nieves, D.; Chaikof, E. L. Collagen and Elastin Biomaterials for the Fabrication of Engineered Living Tissues. ACS Biomater. Sci. Eng. 2017, 3 (5), 694-711.

(7) Hulmes, D. J. S. Building Collagen Molecules, Fibrils, and Suprafibrillar Structures. J. Struct. Biol. 2002, 137 (1-2), 2-10.

(8) Oechsle, A. M.; Landenberger, M.; Gibis, M.; Irmscher, S. B.; Kohlus, R.; Weiss, J. Modulation of Collagen by Addition of Hofmeister Salts. Int. J. Biol. Macromol. 2015, 79, 518-526.

(9) Oechsle, A. M.; Wittmann, X.; Gibis, M.; Kohlus, R.; Weiss, J. Collagen Entanglement Influenced by the Addition of Acids. Eur. Polym. J. 2014, 58, 144-156.

(10) Hayashi, T.; Nagai, Y. Factors Affecting the Interactions of Collagen Molecules as Observed by in Vitro Fibril Formation. J. Biochem. (Tokyo) 1973, 74 (2), 253-262.

(11) Williams, B. R.; Gelman, R. A.; Poppke, D. C.; Piez, K. A. Collagen Fibril Formation. Optimal in Vitro Conditions and Preliminary Kinetic Results. J. Biol. Chem. 1978, 253 (18), 6578-6585.

(12) Wess, T. J. Collagen Fibrillar Structure and Hierarchies. In Collagen - Structure and Mechanics; Fratzl, P.: New York, US., 2008; pp 49-80.

(13) Ramírez-Rodríguez, G. B.; Iafisco, M.; Tampieri, A.; Gómez-Morales, J.; Delgado-López, J. M. PH-Responsive Collagen Fibrillogenesis in Confined Droplets Induced by Vapour Diffusion. J. Mater. Sci. Mater. Med. 2014, 25 (10), 2305-2312.

(14) Besseau, L.; Giraud-Guille, M.-M. Stabilization of Fluid Cholesteric Phases of Collagen to Ordered Gelated Matrices. J. Mol. Biol. 1995, 251 (2), 197-202.

(15) Mosser, G.; Anglo, A.; Helary, C.; Bouligand, Y.; Giraud-Guille, M.-M. Dense Tissuelike Collagen Matrices Formed in Cell-Free Conditions. Matrix Biol. 2006, 25 (1), 3-13.

(16) Gobeaux, F.; Mosser, G.; Anglo, A.; Panine, P.; Davidson, P.; Giraud-Guille, M.-M.; Belamie, E. Fibrillogenesis in Dense Collagen Solutions: A Physicochemical Study. J. Mol. Biol. 2008, 376 (5), 1509-1522.

(17) De Sa Peixoto, P.; Deniset-Besseau, A.; Schmutz, M.; Anglo, A.; Illoul, C.; SchanneKlein, M.-C.; Mosser, G. Achievement of Cornea-like Organizations in Dense Collagen I Solutions: Clues to the Physico-Chemistry of Cornea Morphogenesis. Soft Matter 2013, 9 (47), 11241. 
(18) Mahmoodani, F.; Ardekani, V. S.; See, S. F.; Yusop, S. M.; Babji, A. S. Optimization and Physical Properties of Gelatin Extracted from Pangasius Catfish (Pangasius Sutchi) Bone. J. Food Sci. Technol. 2014, 51 (11), 3104-3113.

(19) Baroudi, I.; Simonnet-Jégat, C.; Roch-Marchal, C.; Leclerc-Laronze, N.; Livage, C.; Martineau, C.; Gervais, C.; Cadot, E.; Carn, F.; Fayolle, B.; et al. Supramolecular Assembly of Gelatin and Inorganic Polyanions: Fine-Tuning the Mechanical Properties of Nanocomposites by Varying Their Composition and Microstructure. Chem. Mater. 2015, 27 (5), 1452-1464.

(20) Radev, L.; Fernandes, M.; Salvado, I.; Kovacheva, D. Organic/Inorganic Bioactive Materials Part III: In Vitro Bioactivity of Gelatin/Silicocarnotite Hybrids. Open Chem. 2009, 7 (4).

(21) Djabourov, M.; Leblond, J.; Papon, P. Gelation of Aqueous Gelatin Solutions. I. Structural Investigation. J. Phys. 1988, 49 (2), 319-332.

(22) Duconseille, A.; Astruc, T.; Quintana, N.; Meersman, F.; Sante-Lhoutellier, V. Gelatin Structure and Composition Linked to Hard Capsule Dissolution: A Review. Food Hydrocoll. 2015, 43, 360-376.

(23) Harrington, W. F.; Rao, N. V. Collagen Structure in Solution. I. Kinetics of Helix Regeneration in Single-Chain Gelatins. Biochemistry (Mosc.) 1970, 9 (19), 3714-3724.

(24) Santoro, M.; Tatara, A. M.; Mikos, A. G. Gelatin Carriers for Drug and Cell Delivery in Tissue Engineering. J. Controlled Release 2014, 190, 210-218.

(25) Foox, M.; Zilberman, M. Drug Delivery from Gelatin-Based Systems. Expert Opin. Drug Deliv. 2015, 12 (9), 1547-1563.

(26) Naahidi, S.; Jafari, M.; Logan, M.; Wang, Y.; Yuan, Y.; Bae, H.; Dixon, B.; Chen, P. Biocompatibility of Hydrogel-Based Scaffolds for Tissue Engineering Applications. Biotechnol. Adv. 2017, 35 (5), 530-544.

(27) Murthy, N. S. Liquid Crystallinity in Collagen Solutions and Magnetic Orientation of Collagen Fibrils. Biopolymers 1984, 23 (7), 1261-1267.

(28) Maeda, H. Observation of Spatially Rhythmic Patterns from Evaporating Collagen Solution Droplets. Langmuir 2000, 16 (26), 9977-9982.

(29) Maeda, H. An Atomic Force Microscopy Study of Ordered Molecular Assemblies and Concentric Ring Patterns from Evaporating Droplets of Collagen Solutions. Langmuir 1999, 15 (24), 8505-8513.

(30) Saeidi, N.; Karmelek, K. P.; Paten, J. A.; Zareian, R.; DiMasi, E.; Ruberti, J. W. Molecular Crowding of Collagen: A Pathway to Produce Highly-Organized Collagenous Structures. Biomaterials 2012, 33 (30), 7366-7374.

(31) Chung, W.-J.; Oh, J.-W.; Kwak, K.; Lee, B. Y.; Meyer, J.; Wang, E.; Hexemer, A.; Lee, S.-W. Biomimetic Self-Templating Supramolecular Structures. Nature 2011, 478 (7369), 364-368.

(32) Tang, M.; Ding, S.; Min, X.; Jiao, Y.; Li, L.; Li, H.; Zhou, C. Collagen Films with Stabilized Liquid Crystalline Phases and Concerns on Osteoblast Behaviors. Mater. Sci. Eng. C 2016, 58, 977-985.

(33) Mitov, M. Cholesteric Liquid Crystals in Living Matter. Soft Matter 2017, 13 (23), 41764209.

(34) Tidu, A.; Ghoubay-Benallaoua, D.; Lynch, B.; Haye, B.; Illoul, C.; Allain, J.-M.; Borderie, V. M.; Mosser, G. Development of Human Corneal Epithelium on Organized Fibrillated Transparent Collagen Matrices Synthesized at High Concentration. Acta 
Biomater. 2015, 22, 50-58.

(35) Ghazanfari, S.; Khademhosseini, A.; Smit, T. H. Mechanisms of Lamellar Collagen Formation in Connective Tissues. Biomaterials 2016, 97, 74-84.

(36) Wiegand, C.; Abel, M.; Ruth, P.; Wilhelms, T.; Schulze, D.; Norgauer, J.; Hipler, U.-C. Effect of the Sterilization Method on the Performance of Collagen Type I on Chronic Wound Parameters in Vitro. J. Biomed. Mater. Res. B Appl. Biomater. 2009, 90B (2), 710719.

(37) Zou, Y.; Broughton, D. L.; Bicker, K. L.; Thompson, P. R.; Lavigne, J. J. Peptide Borono Lectins (PBLs): A New Tool for Glycomics and Cancer Diagnostics. ChemBioChem 2007, 8 (17), 2048-2051.

(38) De Sa Peixoto, P.; Deniset-Besseau, A.; Schanne-Klein, M.-C.; Mosser, G. Quantitative Assessment of Collagen I Liquid Crystal Organizations: Role of Ionic Force and Acidic Solvent, and Evidence of New Phases. Soft Matter 2011, 7 (23), 11203.

(39) Teulon, C.; Tidu, A.; Portier, F.; Mosser, G.; Schanne-Klein, M.-C. Probing the 3D Structure of Cornea-like Collagen Liquid Crystals with Polarization-Resolved SHG Microscopy. Opt. Express 2016, 24 (14), 16084.

(40) Bancelin, S.; Nazac, A.; Ibrahim, B. H.; Dokládal, P.; Decencière, E.; Teig, B.; Haddad, H.; Fernandez, H.; Schanne-Klein, M.-C.; De Martino, A. Determination of Collagen Fiber Orientation in Histological Slides Using Mueller Microscopy and Validation by Second Harmonic Generation Imaging. Opt. Express 2014, 22 (19), 22561.

(41) Deniset-Besseau, A.; De Sa Peixoto, P.; Mosser, G.; Schanne-Klein, M.-C. Nonlinear Optical Imaging of Lyotropic Cholesteric Liquid Crystals. Opt. Express 2010, 18 (2), 1113-1121.

(42) Guo, L.; Colby, R. H.; Lusignan, C. P.; Whitesides, T. H. Kinetics of Triple Helix Formation in Semidilute Gelatin Solutions. Macromolecules 2003, 36 (26), 9999-10008.

(43) Prost, J.; De Gennes, P.-G. Liquid Crystal: Main Types and Properties. In The physics of Liquid Crystals; Prost, J \& De Gennes, P.G.: Oxford, UK, 1993.

(44) Boedtker, H.; Doty, P. A Study of Gelatin Molecules, Aggregates and Gels. J. Phys. Chem. 1954, 58 (11), 968-983.

(45) Miles, C. A.; Burjanadze, T. V.; Bailey, A. J. The Kinetics of the Thermal Denaturation of Collagen in Unrestrained Rat Tail Tendon Determined by Differential Scanning Calorimetry. J. Mol. Biol. 1995, 245 (4), 437-446.

(46) Lorén, N.; Hermansson, A.-M.; Williams, M. A. K.; Lundin, L.; Foster, T. J.; Hubbard, C. D.; Clark, A. H.; Norton, I. T.; Bergström, E. T.; Goodall, D. M. Phase Separation Induced by Conformational Ordering of Gelatin in Gelatin/Maltodextrin Mixtures. Macromolecules 2001, 34 (2), 289-297.

(47) Esquena, J. Water-in-Water (W/W) Emulsions. Curr. Opin. Colloid Interface Sci. 2016, 25, 109-119.

(48) Gobeaux, F.; Belamie, E.; Mosser, G.; Davidson, P.; Panine, P.; Giraud-Guille, M.-M. Cooperative Ordering of Collagen Triple Helices in the Dense State. Langmuir 2007, 23 (11), 6411-6417.

(49) Wang, S.; van Dijk, J. A. P. P.; Odijk, T.; Smit, J. A. M. Depletion-Induced Demixing in Aqueous Protein-Polysaccharide Solutions. Biomacromolecules 2001, 2 (4), 1080-1088.

(50) Edelman, M. W.; van der Linden, E.; de Hoog, E.; Tromp, R. H. Compatibility of Gelatin and Dextran in Aqueous Solution. Biomacromolecules 2001, 2 (4), 1148-1154.

(51) Gobeaux, F.; Belamie, E.; Mosser, G.; Davidson, P.; Asnacios, S. Power Law Rheology 
and Strain-Induced Yielding in Acidic Solutions of Type I-Collagen. Soft Matter 2010, 6 (16), 3769.

(52) Elharfaoui, N.; Djabourov, M.; Babel, W. Molecular Weight Influence on Gelatin Gels: Structure, Enthalpy and Rheology. Macromol. Symp. 2007, 256 (1), 149-157.

(53) Yamashita, Y.; Yanagisawa, M.; Tokita, M. Sol-gel Transition and Phase Separation in Ternary System of Gelatin-Water-poly(Ethylene Glycol) Oligomer. J. Mol. Liq. 2014, 200, 47-51.

(54) Clark, A. H.; Richardson, R. K.; Ross-Murphy, S. B.; Stubbs, J. M. Structural and Mechanical Properties of Agar/Gelatin Co-Gels. Small-Deformation Studies. Macromolecules 1983, 16 (8), 1367-1374.

(55) Varrato, F.; Di Michele, L.; Belushkin, M.; Dorsaz, N.; Nathan, S. H.; Eiser, E.; Foffi, G. Arrested Demixing Opens Route to Bigels. Proc. Natl. Acad. Sci. 2012, 109 (47), 1915519160.

(56) Norton, I. T.; Frith, W. J. Microstructure Design in Mixed Biopolymer Compposites. Food Hydrocoll. 2001, 15, 543-533.

(57) Kozlov, P. V.; Burdygina, G. I. The Structure and Properties of Solid Gelatin and the Principles of Their Modification. 1983, 24, 651-666.

(58) Marchal, A.; Steunou, N.; Mosser, G.; Coradin, T.; Maquet, J.; Livage, J. Assembly of Decavanadate Polyanions by Collagen Helices: Synthesis of an Hybrid InorganicBioorganic Mmaterial. MRS Online Proc. Libr. Arch. 2006, 972.

(59) Eglin, D.; Mosser, G.; Giraud-Guille, M.-M.; Livage, J.; Coradin, T. Type I Collagen, a Versatile Liquid Crystal Biological Template for Silica Structuration from Nano- to Microscopic Scales. Soft Matter 2005, 1 (2), 129.

(60) Coradin, T.; Bah, S.; Livage, J. Gelatine/Silicate Interactions: From Nanoparticles to Composite Gels. Colloids Surf. B Biointerfaces 2004, 35 (1), 53-58. 


\section{Supporting Information}

Title: Stabilization of collagen fibrils by gelatin addition: a study of

dense phases collagen/gelatin

François Portier, Claire Teulon, Agnieszka Nowacka-Perrin, Flavien Gueneau, Marie-Claire Schanne-Klein, and Gervaise Mosser*

\section{Supplementary Methods}

Synthesis of (FITC)-tagged gelatin A.

(FITC)-tagged gelatin A was prepared following the protocol of Zou et al. ${ }^{25} 1.25 \mathrm{~g}$ of gelatin A were solubilized in $250 \mathrm{ml}$ of carbonate buffer $(0.5 \mathrm{M}$ and $\mathrm{pH}=9.5)$ by a moderate heating at $45^{\circ} \mathrm{C}$ for $20 \mathrm{~min}$. Gelatin solution was then filtered (porosity $=0.2$ microns) and $25 \mathrm{~mL}$ of a solution of FITC in ethanol was added ( $\mathrm{w}_{\text {FITC }}=25 \mathrm{mg}$ ). The solution was continuously stirred during 3 hours at $20^{\circ} \mathrm{C}$ prior to an intensive dialysis against acetic acid $(0.5 \mathrm{M})$. Elimination of unreacted FITC was assessed by fluorimetry on the dialysis bath. (FITC)-tagged gelatin solution was stored at $4^{\circ} \mathrm{C}$ and its acid composition was modified through dialysis prior use. The gelatin concentration was determined from the acidic solutions by assessing the amount of hydroxyproline and diluted in consequence.

\section{Supplementary Figures}

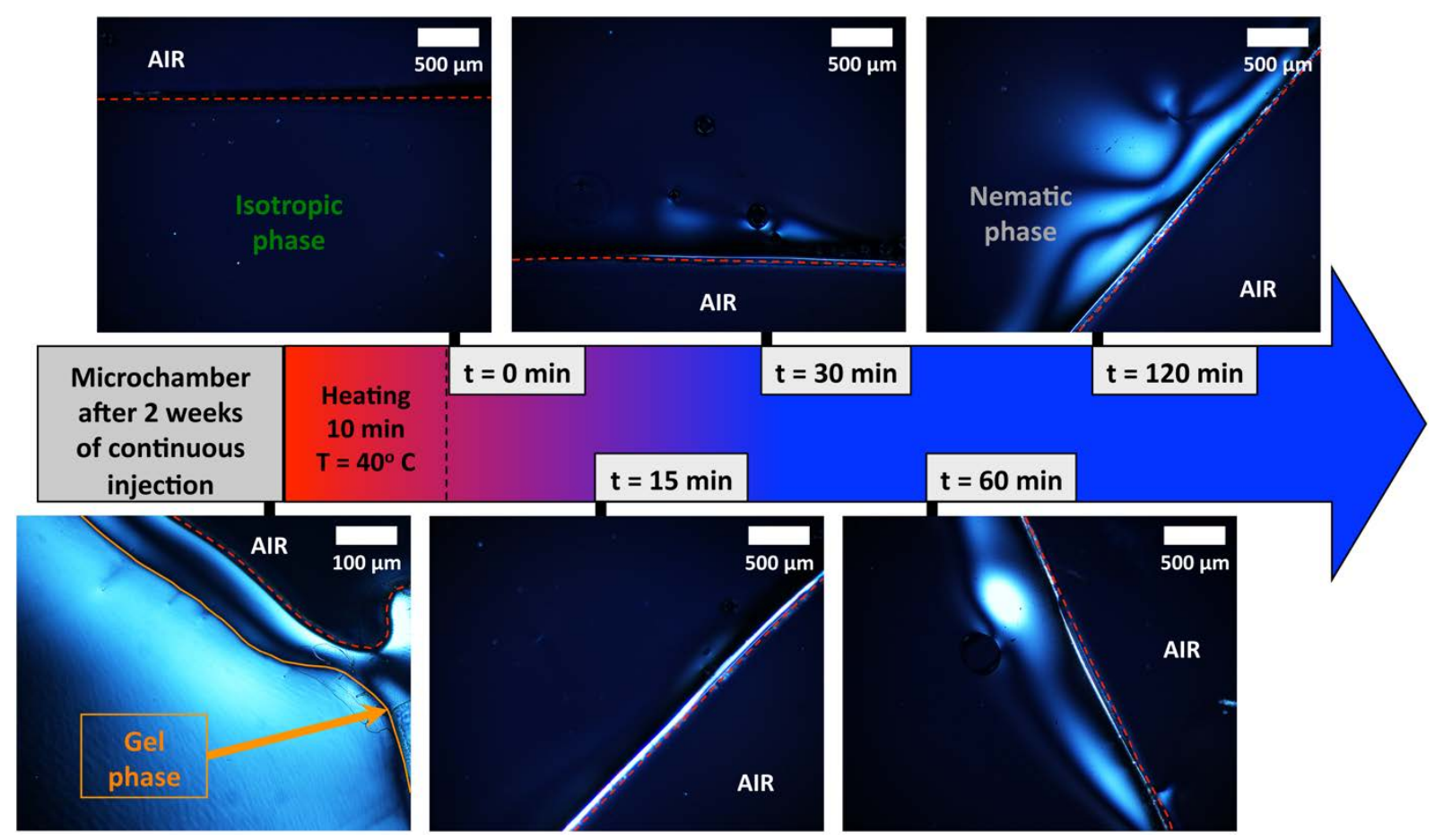


Figure S1: Behavior of a gelatin A mesophase (condition 5 from table S1) after thermal denaturation. Initially, a gelatin mesophase is formed after 2 weeks of continuous injection in a microchamber, as confirmed by PLM. The microchambers is then warmed at $40^{\circ} \mathrm{C}$ for 10 minutes. Right after this treatment, at $\mathrm{t}=0$, the birefringence is completely lost. However, as the solution is cooled, we observed a progressive reappearance of the gelatin birefringence.

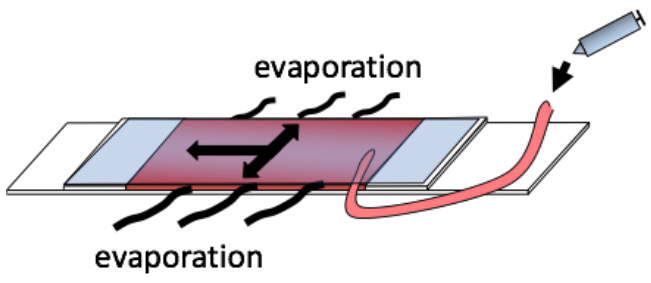

Continuous injection of diluted solution of proteins

Figure S2: Schematic representation of a glass microchamber ${ }^{26}$. Two-sided open glass microchambers were made from microscope glass slides and coverslips with $300 \mu \mathrm{m}$ thick glass spacers. The chambers were filled with protein solutions and kept at $20^{\circ} \mathrm{C}$. Slow and continuous injection of the same solution was used to counteract evaporation and to progressively increase the collagen/gelatin concentration in the chamber. A protein concentration gradient progressively set up between the water/air interface (high concentration) and the center of microchamber (low concentration). The very slow process achieved by this setup was designed to avoid artefacts due to evaporation and gave highly reproducible results. Each experience was repeated at least 3 times. The organization of collagen, correlated with birefringent signals, was directly monitored by PLM and P-SHG within the glass microchambers.

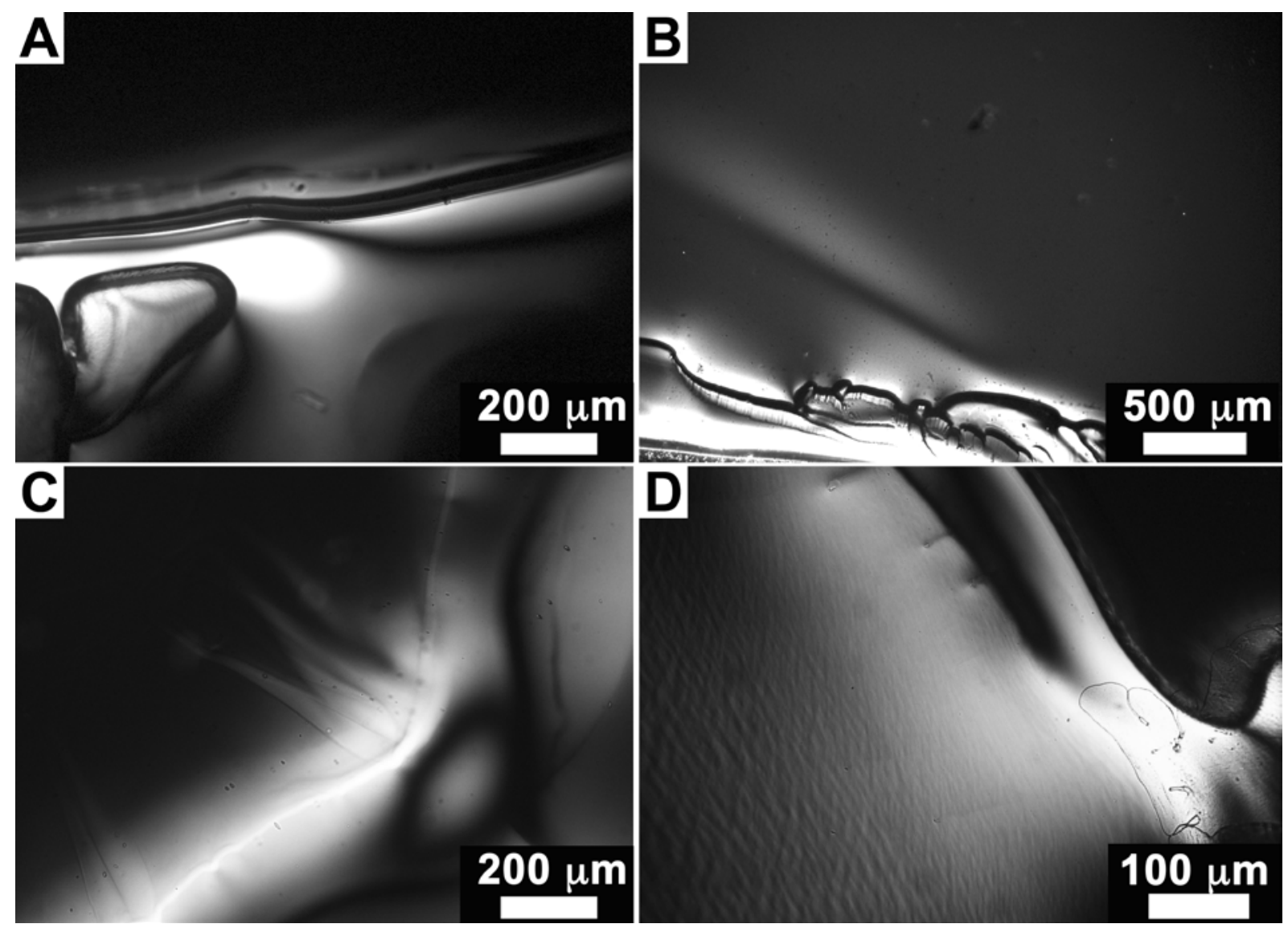

Figure S3: Effect of initial pH and acid type on gelatin A dense phase structure. Observed by PLM after 2 weeks of continuous injection in microchambers: (A \& B) $\mathrm{pH}=2.5$ in $\mathrm{CH}_{3} \mathrm{COOH} 500 \mathrm{mM}$ or $\mathrm{HCl} 3 \mathrm{mM}$ respectively conditions $2 \& 4$ and $(\mathrm{C} \& \mathrm{D}) \mathrm{pH}=3.5$ in $\mathrm{CH}_{3} \mathrm{COOH} 5 \mathrm{mM}$ or $\mathrm{HCl} 0.3 \mathrm{mM}$ respectively conditions $3 \& 5$. Note that $\mathrm{pH}$, we observed different optical signatures mainly in function of $\mathrm{pH}$ (AB/AD) but 
also in function of acid mostly at low $\mathrm{pH}(\mathrm{C} / \mathrm{D})$. The wrinkles observed in $\mathrm{D}$ suggest a possible deviation from the simple nematic phase. Further analysis should be undertaken to fully characterize this phase.

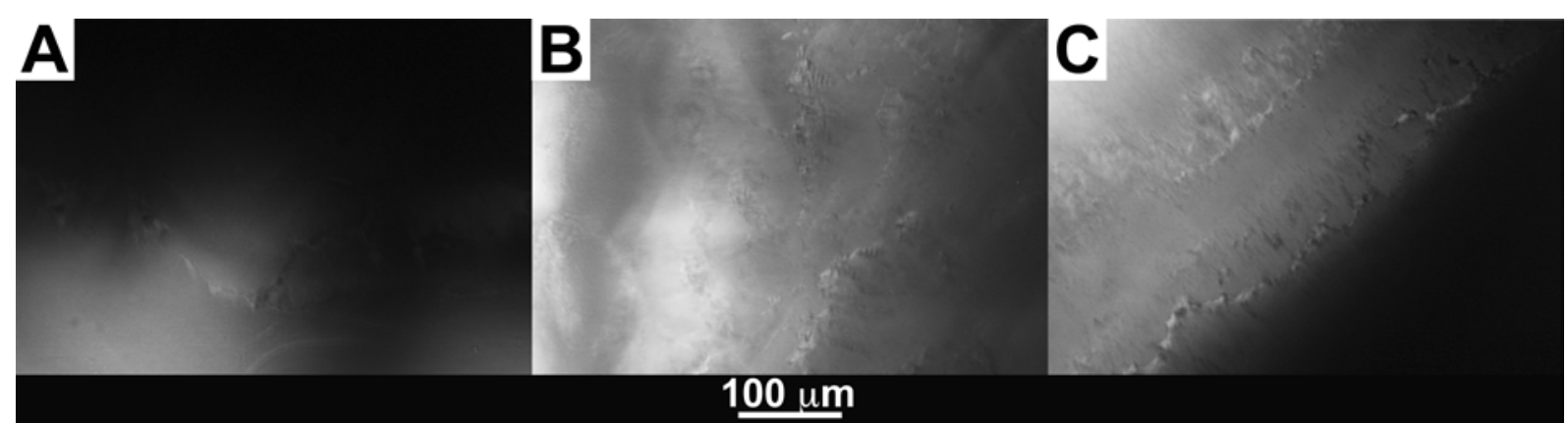

Figure S4: Gelatin A mesophases in presence of collagen at high gelatin/collagen ratios in glassmicrochambers. A, B, C: $75 \%, 90 \%$ and $95 \%$. Due to the high birefringence of the nematic gelatin, the localized biregringence of collagen can be hard to track down. However, the typical striated signature of collagen twisted nematic can be clearly recognized at some places especially in B.

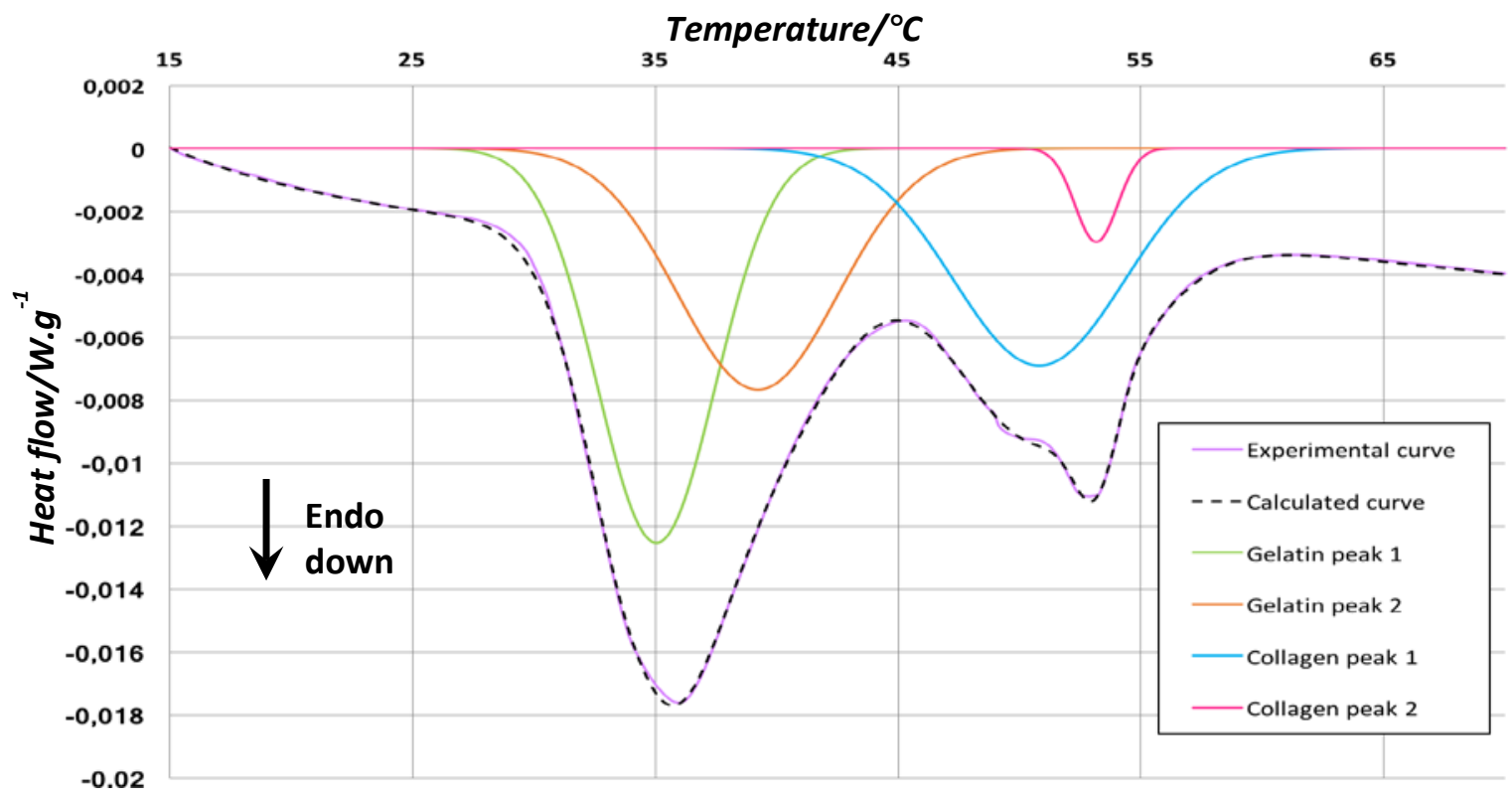

Figure S5: Deconvolution of matrices thermograms. For each thermogram obtained by DSC, a least squares methodology was used to make a numerical fit considering a function given by the sum of four Gaussian functions (corresponding to two contributions for each proteins, collagen and gelatin), and a sigmoid function (reflecting a change in the specific heat of the matrix during the denaturation process). On the graph, above, is represented the thermogram of a matrix m85\% ( Experimental curve » in dark blue on figure 5) and the calculated curve (dotted line in black) that fits for it. The four Gaussian functions that composed this calculated curve are also represented and are then used to determine both temperatures and enthalpies of proteins denaturation. The color code used here for the four Gaussian functions is the same used is the figures $5 \mathrm{~B}$ to $5 \mathrm{E}$ 


\section{Supplementary Tables}

\begin{tabular}{|l|l|l|l|}
\hline \multicolumn{5}{|c|}{ Table S1 } \\
\hline Condition & $\begin{array}{c}\text { C(gelatin) init } \\
{\left[\mathbf{m g ~ m L}^{-1}\right]}\end{array}$ & $\begin{array}{c}\left.\text { C(CH }{ }_{3} \mathbf{C O O H} / \mathbf{H C l}\right) \\
{[\mathbf{m M}]}\end{array}$ & $\mathbf{p H}$ init \\
\hline 1 & 2 & $500 / 0$ & 2.5 \\
\hline 2 & 0.2 & $500 / 0$ & 2.5 \\
\hline 3 & 0.2 & $5 / 0$ & 3.5 \\
\hline 4 & 0.2 & $0 / 3$ & 2.5 \\
\hline 5 & 0.2 & $0 / 0.3$ & 3.5 \\
\hline 6 & 0.2 & $2.5 / 0.15$ & 3.5 \\
\hline
\end{tabular}

Table S1: Composition of the diluted gelatin A solutions before their injection in microchambers. All samples were observed by PLM and P-SHG.

\begin{tabular}{|c|c|c|c|}
\hline \multicolumn{4}{|c|}{ Table S2 } \\
\hline Condition & \% gel & $\%$ col & $\begin{array}{l}\text { C(Proteins) })_{\text {init }} \\
{\left[\mathrm{mg} \mathrm{mL}^{-1}\right]}\end{array}$ \\
\hline g0\% & 0 & 100 & 2 \\
\hline g5\% & 5 & 95 & 2 \\
\hline g15\% & 15 & 85 & 2 \\
\hline g25\% & 25 & 75 & 2 \\
\hline g50\% & 50 & 50 & 2 \\
\hline g75\% & 75 & 25 & 2 \\
\hline g85\% & 85 & 15 & 2 \\
\hline g95\% & 95 & 5 & 2 \\
\hline g100\% & 100 & 0 & 2 \\
\hline
\end{tabular}

Table S2: Composition of the diluted collagen/gelatin solutions before their injection in microchambers. Proteins are solubilized in a mix of acetic $(10 \mathrm{mM})$ and hydrochloric $(0.3 \mathrm{mM})$ acids. All samples were observed by PLM and P-SHG. 


\begin{tabular}{|l|l|l|c|c|}
\hline \multicolumn{5}{|c|}{ Table S3 } \\
\hline Condition & \% gel & \% col & DSC & $\begin{array}{c}\text { MRI, P-SHG, } \\
\text { PLM, TEM }\end{array}$ \\
\hline m0\% & 0 & 100 & $\checkmark$ & $\checkmark$ \\
\hline $\mathbf{m 5 \%}$ & 5 & 95 & $\checkmark$ & - \\
\hline $\mathbf{m 1 5 \%}$ & 15 & 85 & $\checkmark$ & - \\
\hline $\mathbf{m} \mathbf{2 5 \%}$ & 25 & 75 & $\checkmark$ & $\checkmark$ \\
\hline $\mathbf{m 5 0 \%}$ & 50 & 50 & $\checkmark$ & $\checkmark$ \\
\hline $\mathbf{m 7 5 \%}$ & 75 & 25 & $\checkmark$ & $\checkmark$ \\
\hline $\mathbf{m 8 5 \%}$ & 85 & 15 & $\checkmark$ & - \\
\hline $\mathbf{m 9 5 \%}$ & 95 & 5 & $\checkmark$ & - \\
\hline $\mathbf{m 1 0 0 \%}$ & 100 & 0 & $\checkmark$ & $\checkmark$ \\
\hline
\end{tabular}

Table S3: Composition of the collagen/gelatin dense matrices. The total protein concentration was set at 100 mg.mL $\mathrm{m}^{-1}$ by centrifugation/filtration. Proteins are solubilized in a mix of acetic $(10 \mathrm{mM})$ and hydrochloric $(0.3$ $\mathrm{mM}$ ) acids. As indicated in the two last columns, all the samples were analyzed by DSC but only five of them were characterized by the several imaging techniques. 